\title{
Frontières
}

\section{LAPLANTE, Julie, Pouvoir guérir. Médecines autochtones et humanitaires, Québec, Presses de l'Université Laval, coll. "Sociétés, cultures et santé ", 2004, 320 p.}

\section{Gabriel Lefebvre}

Volume 18, numéro 1, automne 2005

URI : https://id.erudit.org/iderudit/1074324ar

DOI : https://doi.org/10.7202/1074324ar

Aller au sommaire du numéro

Éditeur(s)

Université du Québec à Montréal

ISSN

1180-3479 (imprimé)

1916-0976 (numérique)

Découvrir la revue

Citer ce compte rendu

Lefebvre, G. (2005). Compte rendu de [LAPLANTE, Julie, Pouvoir guérir. Médecines autochtones et humanitaires, Québec, Presses de l'Université Laval, coll. « Sociétés, cultures et santé », 2004, 320 p.] Frontières, 18(1), 73-74. https://doi.org/10.7202/1074324ar d'utilisation que vous pouvez consulter en ligne.

https://apropos.erudit.org/fr/usagers/politique-dutilisation/ 


\section{Pouvoir guérir}

Médecines autochtones et humanitaires

Québec, Presses de l'Université Laval, coll. «Sociétés, cultures et santé», 2004, 320 p.

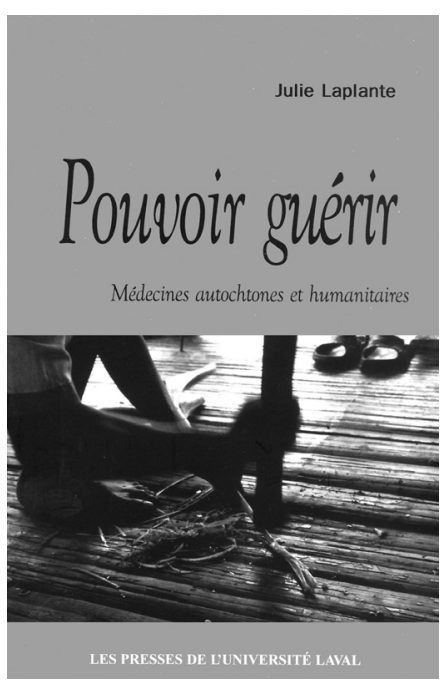

La jeune anthropologue publie ici ses travaux de terrains auprès de populations indigènes d'Amazonie. Ses réflexions s'inscrivent dans le cadre des stratégies de développement mises de l'avant, entre autres, par l'ONG Médecins sans 
frontières (MSF). Cette forme d'humanitarisme d'État, par l'usage de techniques interventionnistes visant le développement de l'autre, tente d'introduire la médecine occidentale scientifique - la biomédecine en territoire autochtone. Ces dernières, cependant, possèdent déjà des savoirs thérapeutiques traditionnels. La mission principale de MSF consiste à instaurer des soins de santé primaires biomédicaux, mais sans nécessairement reconnaître la portée que peuvent avoir les savoirs locaux. Pour Laplante, ce contexte d'intervention fait émerger la problématique des rapports entre savoir et pouvoir aux lieux mêmes où des savoirs thérapeutiques locaux rencontrent ce savoir qui se veut global, la biomédecine. À partir de longs passages sur le terrain, l'auteure cherche à repérer s'il y a transformation, expropriation ou construction de nouvelles formes de savoirs issues de ces rencontres, tout en tenant compte de l'enjeu de la reconnaissance - ou méconnaissance - des savoirs traditionnels.

L'objectif de cette recherche était en outre double: d'une part, découvrir les dynamiques savoir/pouvoir de la rencontre entre savoirs locaux et savoirs cosmopolites; d'autre part, développer des outils anthropologiques pour démystifier les effets de pouvoir associés aux formes de savoirs et pour valoriser les savoirs autochtones locaux. Cette seconde tâche met en relief le rôle de l'anthropologie ainsi que sa portée sociale, que l'auteure explorera en fin de volume en présentant sa démarche réflexive.

Pour accomplir ses objectifs, l'anthropologue a entrepris une approche ethnographique multisite en participant comme chercheuse-anthropologue-volontaire au sein de MSF-Hollande lors de I'intervention de l'organisme au Brésil. Quatre sites ont fait l'objet d'observations et d'analyses: le site MSF international à son centre opérationnel de Hollande et à sa section nationale au Canada; le site national de MSF au Brésil, à Manaus, lieu de coordination des projets locaux avec l'organisme international; le site MSF local à Téfé dans la région du Médio Solimões et d'Atalaise do Norte dans la vallée du Javari en Amazonie; enfin des sites autochtones ciblés par le projet MSF dans le Médio Solimões. L'auteure a examiné, dans les quatre sites, les différents niveaux d'interactions entre les savoirs et les formes de métissage à travers l'axe intervenant biomédical/médicament pharmaceutique et tradipraticien/plante médicinale. À partir de nombreuses situations d'observation participante, de plusieurs séries d'entretiens, et d'analyses des effets de réflexivité de ces deux techniques, I'anthropologue propose ici les résultats de ses recherches.

Son premier chapitre présente le contexte théorique de l'œuvre: approche critique constructiviste en anthropologie médicale, philosophie et sociologie de la connaissance et anthropologie de l'expérience. Le chapitre suivant, quant à lui, esquisse un portrait de la région visée par le terrain et situe globalement les interventions de MSF dans ce territoire.

Dans les chapitres suivants, I'auteure aborde chacun des sites étudiés. L'anthropologue nous explique d'abord comment le site international de MSF-Hollande - et sa branche canadienne - véhicule fidèlement un savoir biomédical humanitaire qui se veut hégémonique. Pour le site de MSF national, I'A. illustre la dynamique entre les savoirs de MSF et ceux des sociétés nationales brésiliennes aussi présentes. Elle y découvre, d'un côté, un système de santé publique avec sa vision humanitaire universelle et, de l'autre, une opposition locale à la globalisation et au problème de la biopiraterie, associée à la lutte revendicatrice pour la reconnaissance autochtone. Le site MSF local, quant à lui, montre bien que les savoirs thérapeutiques sont méconnus dans la pratique des différents intervenants. Malgré les efforts de reconnaissance, le site local maintient le savoir biomédical, alors que le site international se fait neutre pour assurer la diffusion de ses idéaux. Le site national, pris entre les deux, devient le lieu où la lutte autochtone s'affiche comme une exigence de l'identité brésilienne, mais sans porter concrètement le contenu des savoirs traditionnels concernés.

Les différents sites autochtones témoignent beaucoup mieux des effets de la rencontre et de l'enchevêtrement des savoirs. L'auteure explore l'intégration des soins biomédicaux et leur interaction avec les savoirs locaux, en présentant les acteurs et leur usage des substances, pour sept sites distincts, chacun manifestant une façon singulière de marier les savoirs. Parfois, I'un des savoirs est ignoré, caché; parfois, l'autre est survalorisé. Le septième site, celui de la population MadijasKulinas, fait l'objet d'un chapitre à part, car il décrit le cas le plus marqué de négociation, en cours, des frontières entre les savoirs. L'anthropologue y repérera des savoirs non seulement en collaboration ou en confrontation, mais interagissant de sorte que des initiatives et une pensée novatrice en émergent. D'un site à l'autre, les savoirs sont liés différemment selon les acteurs, mais le projet commun demeure: celui de guérir.

Au chapitre suivant, l'auteure tente de faire sens en observant les différents contextes, montrant comment le lieu d'accueil modifie les savoirs afin que leurs détenteurs conservent un pouvoir de dire et d'agir. Il y a bien un métissage local, un réel lien de continuité entre les savoirs. On n'a plus affaire à des savoirs locaux ni globaux, mais à un enchevêtrement des deux, variant d'un endroit à l'autre.

L'ouvrage se termine sur des considérations méthodologiques. L'auteure explore la portée de la réflexivité comme concept, technique de recherche et moyen d'appliquer I'anthropologie. Elle pose que la réflexivité ouvre à des actions constructives qui peuvent contribuer aux métissages des savoirs et identifier des liens de continuité, plutôt qu'à simplement noter des oppositions ou ruptures. C'était d'ailleurs là la tâche qu'elle s'était donnée lors de ses séjours sur le terrain.

Malgré ces derniers, le lecteur reste sur sa faim. En effet, l'ouvrage fait peu de place à la modélisation ou à la théorisation des rapports entre les savoirs décrits. Cela tient sans doute au fait que l'analyse de I'auteure tente de dresser un portrait général à partie de rencontres très divergentes. II semble difficile de dégager des lois ou des modèles lorsque chaque site autochtone manifeste un métissage unique. En revanche, Pouvoir guérir propose de riches et abondantes descriptions, construites dans le souci du détail et de la nuance, nous présentant avec brio la portée réelle des savoirs sur chaque site. L'étonnante complexité des rapports décrits témoigne de la délicatesse de la tâche et de l'adresse nécessaire pour parvenir à en rendre compte. L'auteure éprouve par ailleurs quelques difficultés en ce qui a trait au style d'écriture, mais elle compense par la justice que rend son exposé aux réalités des divers sites. Elle y parvient sans avoir à prendre parti pour l'un ou l'autre des différents camps en présence, qui radicalisent la situation dans leur discours.

Gabriel Lefebvre 